\title{
PASCHEN DECREMENTS IN Be STARS
}

\author{
D. BRIOT \\ Observatoire de Paris, France
}

\begin{abstract}
We searched for the general properties of the Be stars with Paschen emission lines. First, we obtained a relation between the infrared excess of the Be stars and the presence of emission in the Paschen lines. Until now, these emission excesses could be related to no physical characteristics of classical Be stars. Then, the measures of Paschen decrements of 12 stars whose spectral types range from $\mathrm{B} 0 \mathrm{e}$ to $\mathrm{B} 5 \mathrm{e}$ allowed us to check several theoretical calculations about the formation of emission lines in the envelopes of Be stars. Thus we can see the prominent part played by the electronic collisions in the Sobolev theory. Indeed, only with calculations taking the electronic collisions into account, can we obtain theoretical values agreeing with both measured Paschen and Balmer decrements for the hottest stars of our sample. However, no theoretical values agree with the observed decrements for the cooler stars.
\end{abstract}

